\title{
Konträre wissenschaftliche Perspektiven in der Erforschung schulischen emotionalen und sozialen Lernens: Wie Synergien entstehen können
}

\author{
Joachim Bröcher \\ Europa-Universität Flensburg
}

\begin{abstract}
Zusammenfassung: In der deutschen Wissenschaftslandschaft des schulbezogenen Förderschwerpunkts der emotionalen und sozialen Entwicklung stehen sich derzeit vor allem evidenzbasierte, datenbasierte Modelle der Verhaltenssteuerung und gesellschaftskritische, qualitative, subjektzentrierte Ansätze und Diskurse gegenüber. Der Beitrag fasst die seit den 8 oer Jahren vorherrschenden Themen und Trends zusammen, setzt diese in Beziehung zu internationalen Entwicklungen und lotet dann Möglichkeiten aus, wie die Wissenschafts-Communities mit ihren unterschiedlichen Perspektiven in kooperativen Forschungsteams arbeiten könnten, um gemeinsam wissenschaftliches Neuland zu erobern. Das speziell für inklusive Schulen entwickelte Modell School-wide Positive Behavior Support bildet dabei den Ausgangspunkt.
\end{abstract}

Schlüsselbegriffe: Emotionales und soziales Lernen, wissenschaftstheoretische Paradigmen, School-wide Positive Behavior Support

\begin{abstract}
How Synergies Can be Created Regarding the Contrary Perspectives in the Field of Research of Emotional and Social Learning in Schools

Summary: In the German academic landscape of the school-related research of emotional and social skills development, evidence-based, data-based models of behavioral control and socially critical, qualitative, subject-centred approaches and discourses are currently confronted with each other. This article summarizes the topics and trends that have prevailed since the 1980 s, relates them to international developments, and then explores ways in which the scientific communities with their different perspectives could work in cooperative research teams to jointly conquer new scientific territory. The School-wide Positive Behavior Support model, specially developed for inclusive schools, might be a starting point.
\end{abstract}

Keywords: Emotional and social learning, scientific paradigms, School-wide Positive Behavior Support

\section{Modellentwicklungen in den 8oer- und goer-Jahren}

Bezogen auf den schulischen Förderschwerpunkt der emotionalen und sozialen Entwicklung steht uns ein über mehrere Fachdisziplinen verteiltes, breit gefächertes Erklärungs-, Re- flexions- und Handlungswissen zur Verfügung. Unter den Akteur/innen, die dieses Wissen aus unterschiedlichen Theoriewelten und Paradigmen heraus entwickeln, besteht teils kein Konsens, was die Wege der Wissensproduktion und die Qualität dieses Wissens betrifft. 
Die pädagogischen Konzepte wurden zunächst entlang der großen psychologischen Schulen entwickelt, d.h. der Psychoanalyse, des Behaviorismus, der Humanistischen Psychologie und ihren Fortführungen, und arbeiteten entsprechend teils mit konträren Menschenund Gesellschaftsbildern. Ebenfalls von Bedeutung waren handlungs- und erlebnisorientierte Strömungen der Reformpädagogik, auch entwicklungspsychologische Ansätze, zentriert um Spiel und symbolische Gestaltung, ferner gesellschaftskritische Theorien, in denen soziale Benachteiligung thematisiert wurde. In den 1990er-Jahren fächerten sich die Zugänge weiter auf. Sozialökologischer Ansatz, Bewältigungsforschung, Systemtheorie, Konstruktivismus, Lösungsorientierung und Resilienz waren jetzt die Themen.

\section{Themen nach der Jahrtausendwende}

Mit dem Aufkommen von Leistungsvergleichen, externen Schulevaluationen, Bildungsstandards und Kompetenzorientierung ab der Jahrtausendwende ging es mehr um das Etablieren von einheitlichen, schulübergreifenden Konzepten. Zugleich gewannen die Themen Heterogenität und Inklusion an Bedeutung, getragen von politischem Rückenwind, in der Folge auch Co-Teaching, Schulprogramm und Schulentwicklung, in geringerem Umfang auch Fragen der schulischen Organisations- und Führungskultur. Das Trainingsraum-Programm, ein US-amerikanischer Import, erreichte als erstes schulweites Programm der Verhaltenssteuerung hohe Popularität. Das Programm versprach den vielen jetzt inklusiv arbeitenden Schulen schnelle Lösungen, obwohl es außer der Einführung von strengen Verhaltensregeln keinerlei substanzielle Veränderungen des Unterrichts selbst in Betracht zieht und allein auf eine außerhalb der Klasse zu regelnde Verhaltensanpassung setzt.

\section{Aktuell: Evidenzbasiert-quantitativ vs. gesellschaftskritisch-qualitativ}

In der Gegenwart zeichnen sich in unserem Fach vor allem zwei Strömungen ab. Auf der einen Seite existiert eine, in sich wiederum heterogene, gesellschaftskritisch orientierte Community, gespeist aus kritischer Theorie, Sozialphilosophie, Anerkennungstheorie, Machtkritik, Gender- und Queer Studies, Disability Studies, Biographieforschung, Sozial- und Kulturgeographie, Cultural Studies, Media Studies oder Critical Discourse Analysis. Forschung ist hier zumeist qualitativ fundiert und arbeitet Subjektperspektiven heraus. Teils handelt es sich um konzeptionelle oder historisch-hermeneutische Ansätze. Menschenbild und Bildungsideal sind an Freiheit, Emanzipation und Solidaritätsfähigkeit orientiert. Im Umgang mit den pädagogischen Zielgruppen werden sprachliche Sensibilität, De-Etikettierung und De-Kategorisierung eingefordert. Das Erheben von Daten durch eine klinisch-psychologisch fundierte Diagnostik und Formen der apparativen Systemsteuerung werden von hier aus kritisch gesehen.

Auf der anderen Seite hat sich eine Community in der Forschung und Lehre unseres Faches etabliert, die an quantitativ-statistischen, evidenzbasierten Maßstäben orientiert ist, in Fortführung des sich seit den 60er Jahren konstituierenden behavioristisch-lerntheoretischen Paradigmas. Behavior Rating, Behavior Management, RTI-Modelle oder Verlaufsdiagnostik sind hier die Schlüsselkonzepte. Diese Strömung erweist sich als kompatibel mit der testund datenbasierten Steuerung von Bildungssystemen.

\section{Themen und Trends auf internationaler Ebene}

Aufschlussreich ist an dieser Stelle ein vergleichender Blick in die Vereinigten Staaten, um zu sehen, welche Entwicklungen dort in den 
führenden internationalen Fachgesellschaften sichtbar werden. Die Association for Childhood Education International (ACEI) folgt etwa einer ausgesprochen politischen Agenda. Es wird eine Vielzahl an Projekten in allen Erdteilen unterstützt. Das Wissenschaftsverständnis ist breit, und vor allem: wertegeleitet. Armut und Gewalt soll begegnet werden durch Bildung für alle und Chancengleichheit. Der Council for Children with Behavioral Disorders (CCBD) ist dagegen allein auf die Generierung und Verbreitung von evidenzbasierten, verhaltensbezogenen Interventionen fokussiert. Dasselbe gilt für die Association for Positive Behavior Support (APBS). Zugleich ist APBS die internationale Plattform, auf der School-wide Positive Behavior Support (PBIS) entwickelt wird, ein System zur Prävention und Intervention bei schulischen Verhaltensproblemen, welches ineinander verschränkte pädagogische, didaktische, sozialpädagogische und interprofessionelle Komponenten enthält. Der Association for Experiential Education (AEE) geht es dagegen um soziales und persönliches Lernen durch Erlebnis und Erfahrung, bevorzugt bei Gruppenaktivitäten in der Natur. Das Wissenschaftsverständnis ist breit und schließt quantitative wie qualitative und gesellschaftskritische Zugänge ein. Die Urban Affairs Association (UAA) schließlich lenkt den Blick auf die sozial- und humangeographischen Räume, in denen Menschen leben. Themen sind hier etwa Segregation, Armut oder eingeschränkte Lebens- und Partizipationsmöglichkeiten in ihrer Rückwirkung auf die Sozialisationsverläufe von Heranwachsenden. Eine weitere Richtung mit starker Repräsentanz im schulischen und universitären Bildungssystem Nordamerikas ist Teaching for Social Justice, eine politisch motivierte Pädagogik, deren Wurzeln bis zur Bürgerrechtsbewegung zurückreichen. Forschungsarbeiten sind hier oftmals qualitativ ausgerichtet. Schlüsselthemen sind Student Voice, Partizipation oder die Rolle der sozialen Communities.
Die gesellschaftskritischen Strömungen finden sich in Deutschland wie auch in den USA gleichermaßen. Das Thema Experiential Education wird dagegen in den deutschen Abteilungen des Faches derzeit kaum abgebildet. Dafür bestehen rege transatlantische Austauschbeziehungen zwischen der deutschen und der amerikanischen Behavior-Community. Was passiert darüber hinaus in Europa? Während in den Niederlanden und den skandinavischen Ländern seit einigen Jahren mit School-wide Positive Behavior Support (z. B. Sailor, Dunlap, Sugai \& Horner, 2009) experimentiert wird, steht in Großbritannien nach wie vor die auch im deutschsprachigen Raum geschätzte Bindungstheorie hoch im Kurs. Die britischen Nurture Groups, als pädagogische Anwendungen der Bindungstheorie im Schulkontext, haben sich allerdings bisher in Deutschland kaum durchsetzen können. Ein weiterer wichtiger Impuls aus Großbritannien, schauen wir etwa auf die politische und pädagogische Arbeit der Social, Emotional and Behavioral Difficulties Association (SEBDA) ist die Etablierung von kooperativen Beziehungen zwischen Schule und Community, wenn in der Schule die emotionale und soziale Förderung von Kindern und Jugendlichen gelingen soll. SEBDA integriert wissenschaftliche Ansätze und Methoden in der vollen Breite.

\section{Trotz aller Gegensätze: Synergien wären möglich}

Zurück zu Deutschland: Bieten sich bei den aufgezeigten Gegensätzen in der Herangehensweise, die sich vereinfachend auf die Formel „Wirksamkeitsforschung versus Gesellschaftskritik“" (Laubenstein \& Scheer, 2017) bringen ließen, auch Synergien? Gibt es einen gemeinsamen Nenner? Lassen sich Datenanalytiker/innen, gesellschaftspolitische Aktivist/innen und philosophisch-soziologische Denker/innen an einen Tisch bringen? Ließen sie sich gar mit ihren unterschiedlichen Perspektiven in kooperative Forschungsteams integrieren? Auch Grosche (2017) hat bereits in diese Richtung vorgedacht. 
Gute Chancen, sich als übergreifender Handlungsrahmen durchzusetzen, dürfte derzeit School-wide Positive Behavior Support besitzen. Das Modell punktet mit seiner Betonung der Prävention, mit dem Optimismus, dass sich pro-soziales Verhalten systematisch vermitteln lässt, und mit seiner durchgegliederten Systematik an evidenzbasierten Interventionen. Im Hinblick auf den Brückenschlag zwischen den einander gegenüberstehenden wissenschaftlichen Communities zeigen sich Synergien etwa bei Check \& Connect. Diese Intervention schlägt den Bogen von der Pflege der pädagogischen Beziehung zu Heranwachsenden mit schulvermeidendem Muster bis hin zu einem systematischen, datenbasierten Monitoring. Dass die Intervention Choice-Making in der pädagogisch-didaktischen Arbeit Lernwiderstand abbaut und so zur Verhaltensverbesserung beiträgt, müsste doch auch eher emanzipatorisch denkenden Lehrer/innen entgegenkommen. Die Intervention Opportunities-to-Respond könnte Anhänger/innen der Beziehungspädagogik zumindest partiell für PBIS interessieren. Im Rahmen von PBIS werden nun mithilfe von diagnostischen Instrumenten drei Gruppen von Schüler/innen an einer Schule gebildet: Die größte, unauffällige Gruppe, mit der generell im Sinne emotionalsozialer Förderung präventiv gearbeitet wird (hier wird von „Tier I“ oder Interventionsebene I gesprochen, vgl. Sailor et al., 2009), eine kleinere Gruppe (Tier II oder Ebene II), in der Risiken hinsichtlich der emotionalen und sozialen Entwicklung gesehen und gezielte, jedoch standardisierte Interventionen eingesetzt werden, und eine in der Regel noch kleinere Gruppe von Schüler/innen mit einem hohen Risiko (Tier III), wo individualisierte verhaltensbezogene Interventionen stattfinden. Doch wollen wir uns wirklich auf Tier III, d. h. der Ebene, wo die Arbeit mit den Heranwachsenden stattfindet, die als wirklich herausfordernd eingestuft worden sind, allein auf funktionsbasierte Analysen und Interventionen beschränken lassen? Warum sollen hier keine
Hip-Hop-Projekte laufen? Warum sollen erfahrene Lehrer/innen hier nicht mit den Schüler/innen über ihre Lebensfragen philosophieren? Weil das so schwer zu operationalisieren und zu überprüfen ist? Die Schwäche von PBIS ist, dass es bislang keine Komponenten beinhaltet, um unter die Oberfläche des Verhaltens zu gelangen und auch dort anzusetzen. Und: Passt das Good Behavior Game wirklich zu einem freiheitlichen Bildungsideal?

Schulentwicklungsworkshops (vgl. Bröcher, 2015) haben auch gezeigt, dass es in Deutschland vermutlich nicht gelingen wird, PBIS in der stringenten amerikanischen Reinform umzusetzen. Die in den hiesigen inklusiven Grund- und Sekundarschulen anzutreffende kollegiale Autonomie wird dies verhindern. Schulen, die sich als lernende Organisation verstehen, nehmen sich auch den Raum für eine kontinuierliche Prozessentwicklung und beständige Kurskorrektur. Da kann es auf die Dauer nichts Festes oder Starres geben. Die Lehrer/innen und Sozialpädagog/innen in den Schulen wollen das System, wenn sie es übernehmen sollen, variieren und mit anderen, aus ihrer Sicht bewährten Handlungsansätzen kombinieren oder ergänzen.

\section{Ein Denken in offenen, variablen Systemen wäre die Voraussetzung}

Wir brauchen daher offene Systeme, die zu unserer pluralistischen, sich ständig verändernden Gesellschaft passen. Machten wir aus PBIS also ein variables System, das die Eigeninitiative der Akteur/innen vor Ort in den Schulen wie auch Formen und Ergebnisse qualitativer, historisch-hermeneutischer und gesellschaftskritischer Forschung integriert, dann wären wir einen großen Schritt weiter. So würde das ganze Modell auch selbstbestimmter und authentischer, denn auch die Schüler/innen wollen ihre Stimme einbringen. Die verhaltens- 
orientierten Datenanalytiker/innen könnten freilich darauf achten, dass die pädagogischen Prozesse nicht ins Ungefähre abdriften, was teils eine Schwäche gesellschaftskritischer und qualitativ fundierter Modelle sein kann. Sich mit der bewährten Student Risk Screening Scale einen Überblick über die Problempotenziale einer Schule zu verschaffen, müsste nicht gleich mit dem Big-Data-Vorwurf belegt werden, wenn sonst Zurückhaltung beim Erheben von Daten geübt würde.

Die Teams von Forscher/innen, die solche Schulen aus ihren verschiedenen Perspektiven begleiten, würden dann methodisch flexibel und theoretisch explorativ auf die sich stellenden Fragen reagieren. Das Good Behavior Game könnte dann etwa den Charakter einer Selbsterfahrungsübung haben, auf die sich Schüler/innen freiwillig einlassen und über die im Anschluss reflektiert würde. Wie konstituiert sich in den verschiedenen Phasen des Spiels die Macht und wie erleben das die Beteiligten? Das sind die entscheidenden Fragen, wenn wir alle miteinander etwas über die in unseren Gesellschaften zunehmenden Steuerungsmechanismen lernen und zugleich emotionale und soziale Kompetenzen aufbauen wollen. Oder auf Tier III ausgehend von Sartre mit einem Jugendlichen über die Faktizität, also das derzeit in seinem Leben Gegebene, sprechen und überlegen, was Transzendenz für ihn sein könnte, im Sinne des Horizonts, in den hinein er sich entwickeln möchte. Tier III würde dann mit enormer Energie aufgeladen. Auch Kuhn (2012) rät uns ja zu Abweichungen von den tradierten Wegen, wenn wir wissenschaftliches Neuland entdecken wollen. Auch könnte das Konzept der Resonanz (Rosa, 2019), bei dem es um das Herstellen von lebendigen Austauschbeziehungen geht, in den kooperativ mit den Lernenden zu entwerfenden Lernräumen eine wesentliche Rolle spielen, wie auch in den kommunikativen Beziehungen zwischen den Akteur/innen aus den sich bisher eher resonanzarm gegenüberstehenden Forschungs-Communities.

\section{Literatur}

Bröcher, J. (2015). Implementing School-Wide Positive Behavioral Interventions and Supports (PBIS) in German schools: The challenge of knowledge politics, education cultures and teacher perspectives. In B. Higgins (ed.), Goal Setting and Personal Development: Teachers'Perspectives, Behavioral Strategies and Impact on Performance, 101-151. New York: Nova Science Publishers.

Grosche, M. (2017). Brücken bauen, statt einreißen! Introspektion der quantitativ-empirischen Sonderpädagogik zur Ermöglichung einer interund transdiskursiven Zusammenarbeit. In D. Laubenstein \& D. Scheer (Hrsg.), Sonderpädagogik zwischen Wirksamkeitsforschung und Gesellschaftskritik, 41-62. Bad Heilbrunn: Klinkhardt.

Kuhn, T. S. (2012). The Structure of Scientific Revolutions. $4^{\text {th }}$ ed. Chicago, London: The University of Chicago Press. https://doi.org/10.7208/chica go/9780226458144.001.0001

Laubenstein, D. \& Scheer, D. (2017). Sonderpädagogik zwischen Wirksamkeitsforschung und Gesellschaftskritik. Bad Heilbrunn: Klinkhardt.

Rosa, H. (2019). Resonanz. Eine Soziologie der Weltbeziehung. Berlin: Suhrkamp.

Sailor, W., Dunlap, G., Sugai, G. \& Horner, R. (eds.) (2009). Handbook of Positive Behavior Support. New York: Springer. https://doi.org/10.1007/97 8-0-387-09632-2

\section{Anschrift des Autors}

\section{Prof. Dr. Joachim Bröcher}

Europa-Universität Flensburg

Institut für Sonderpädagogik

Auf dem Campus 1 a

D-24943 Flensburg

E-Mail: joachim.broecher@uni-flensburg.de 